\title{
Comparing various functions of the divisors of an integer in different residue classes
}

\author{
ILDIKÓ KÉZÉR
}

\begin{abstract}
The main goal of this paper is to investigate some problems related to the distribution of the divisors of a number in different residue classes. We study these questions modulo 3, and use mostly just elementary number theory. In some special cases, we demonstrate how this problem is related to other fields of maths, especially to combinatorics. Since the author is also a secondary school teacher, we use elementary methods that can be discussed in secondary school, mainly within the framework of group study sessions or in special maths classes. We do think that the investigation of these types of questions can motivate children to find their own way to create their own questions, and to get a deeper insight into problem solving by these experimentations.
\end{abstract}

Key words and phrases: number and sum of divisors, residue classes.

ZDM Subject Classification: F60.

\section{Introduction}

Examining the ratio of the sums of even and odd (positive) divisors of a given $n$, it can be easily seen that for $n=2^{\gamma} \cdot \prod_{i} p_{i}{ }^{\alpha_{i}}$, where the primes $p_{i} \equiv 1(\bmod 2)$, this ratio is

$$
\frac{\sum_{\substack{x \mid n \\ x \equiv 0(2)}} x}{\sum_{\substack{x \mid n \\ x \equiv 1(2)}} x}=2^{\gamma+1}-2 .
$$

Copyright (C) 2016 by University of Debrecen 
Similarly, if $n=3^{\gamma} \cdot \prod_{i} q_{i}{ }^{\alpha_{i}} \cdot \prod_{j} p_{j}{ }^{\beta_{j}}$, where the primes $q_{i} \equiv-1(\bmod 3), p_{j} \equiv 1$ $(\bmod 3)$ then

Also, for any prime $p$, we have

$$
\frac{\sum_{\substack{x \mid n \\ x \equiv 0(3)}} x}{\sum_{\substack{x \mid n \\ x \equiv \pm 1(3)}} x}=\frac{3^{\gamma+1}-3}{2}
$$

$$
\frac{\sum_{\substack{x>n \\ x \equiv 0(p)}} x}{\sum_{\substack{x \mid n \\ x \neq 0(p)}} x}=\frac{p^{\gamma+1}-p}{p-1}
$$

where $\gamma$ is the exponent of $p$ in the standard form of $n$.

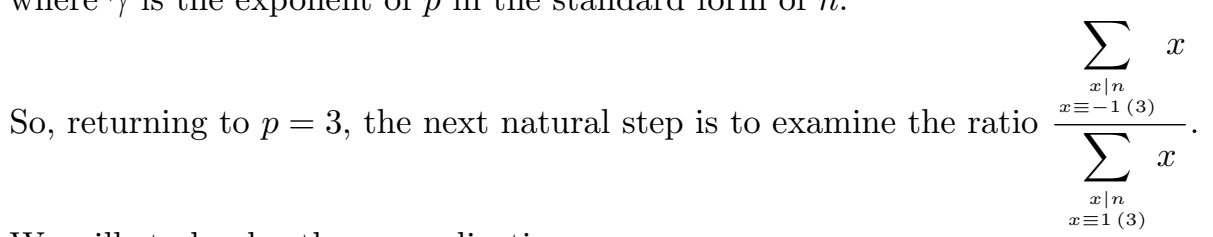

We will study also the generalizations

$$
R_{f}(n)=\frac{\sum_{\substack{x \neq n \\ x=-1(3)}} f(x)}{\sum_{\substack{x \mid n \\ x \equiv 1(3)}} f(x)} \text { and } D_{f}(n)=\sum_{\substack{x \mid n \\ x \equiv 1(3)}} f(x)-\sum_{\substack{x \mid n \\ x \equiv-1(3)}} f(x)
$$

where $f$ is a given function, i.e. we examine the ratio and the difference of these sums for some given functions $f$, such as $f(x)=1, f(x)=x$, and $f(x)=\frac{1}{x}$. It is clear that if

$$
n=3^{\gamma} \cdot \underbrace{\prod_{i} q_{i}{ }^{\alpha_{i}}}_{A} \cdot \underbrace{\prod_{j} p_{j}{ }^{\beta_{j}}}_{B},
$$

where $q_{i} \equiv-1(\bmod 3), p_{j} \equiv 1(\bmod 3)$, then for any $x \equiv \pm 1(\bmod 3)$, we have $x|n \Leftrightarrow x| A B$. 
Therefore it is enough to investigate the ratio $R_{f}(n)$ and the difference $D_{f}(n)$ for numbers $n$ not divisible by 3 , i.e. of the form

$$
n=\underbrace{\prod_{i} q_{i}{ }^{\alpha_{i}}}_{A} \cdot \underbrace{\prod_{j} p_{j}{ }^{\beta_{j}}}_{B},
$$

where $q_{i} \equiv-1(\bmod 3), p_{j} \equiv 1(\bmod 3)$.

Also, the next statements are straightforward:

LEMMA 1. Let $f$ be a multiplicative function, i.e. $(a, b)=1$ implies $f(a b)=$ $f(a) f(b)$. If

$$
n=\underbrace{\prod_{i} q_{i}{ }^{\alpha_{i}}}_{A} \cdot \underbrace{\prod_{j} p_{j}{ }^{\beta_{j}}}_{B},
$$

where $q_{i} \equiv-1(\bmod 3), p_{j} \equiv 1(\bmod 3)$, then

$$
R_{f}(n)=\frac{\sum_{\substack{x \mid A \\ x \equiv-1(3)}} f(x)}{\sum_{\substack{x \mid A \\ x \equiv 1(3)}} f(x)}
$$

and

$$
D_{f}(n)=\left(\sum_{x \mid B} f(x)\right) \cdot\left(\sum_{\substack{x \mid A \\ x \equiv 1(3)}} f(x)-\sum_{\substack{x \mid A \\ x \equiv-1(3)}} f(x)\right) .
$$

LEMma 2. If $f(x)>0$, then $R_{f}(n)=1 \Leftrightarrow D_{f}(n)=0, \quad R_{f}(n)>1 \Leftrightarrow$ $D_{f}(n)<0$, and $R_{f}(n)<1 \Leftrightarrow D_{f}(n)>0$.

Comparing the number of divisors in different residue classes

Let $f(x)=1$, so we study the ratio $R_{1}(n)$ and the difference $D_{1}(n)$ of the number of divisors $d$ of $n$ for which $d \equiv \pm 1(\bmod 3)$. 
Lemma 3 . If $n=\prod_{i} q_{i}{ }^{\alpha_{i}}$, where $q_{i} \equiv-1(\bmod 3)$, then

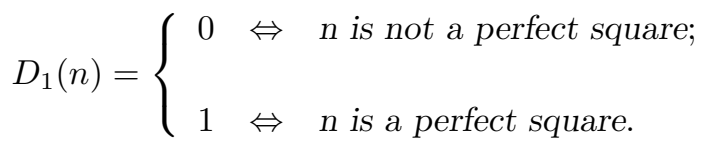

Proof. If $n$ is not a perfect square, then there exists a prime $q_{j}$ with an odd exponent $\alpha_{j}$. We start to list the divisors of $n$ by pairing the powers of $q_{j}$, so that the two elements of a pair belong to different residue classes as the table shows below:

$$
\begin{array}{cc}
\equiv 1(3) & \equiv-1(3) \\
1 & q_{j} \\
q_{j}^{2} & q_{j}^{3} \\
\vdots & \vdots \\
q_{j}^{\alpha_{j}-1} & q_{j}^{\alpha_{j}}
\end{array}
$$

If we have another prime factor $q_{m}$, then we can form new pairs by multiplying the previous table with every power of $q_{m}$ (the parity and size of $\alpha_{m}$ are irrelevant):

$$
\begin{aligned}
& \equiv 1(3) \equiv-1(3) \quad \equiv 1(3) \equiv-1(3) \quad \equiv 1(3) \equiv-1(3) \\
& \begin{array}{llllll}
1 & q_{j} & q_{m} q_{j} & q_{m} & q_{m}^{2} & q_{m}^{2} q_{j}
\end{array} \\
& q_{j}^{2} \quad q_{j}^{3} \quad q_{m} q_{j}^{3} \quad q_{m} q_{j}^{2} \quad q_{m}^{2} q_{j}^{2} \quad q_{m}^{2} q_{j}^{3}
\end{aligned}
$$

\begin{tabular}{|c|c|c|c|c|c|}
\hline$\equiv 1(3)$ & $\equiv-1(3)$ & $\equiv 1(3)$ & $\equiv-1(3)$ & $\equiv 1(3)$ & $\equiv-1(3)$ \\
\hline 1 & $q_{1}$ & $q_{2} q_{1}$ & $q_{2}$ & $q_{2}^{2}$ & $q_{2}^{2} q_{1}$ \\
\hline$q_{1}^{2}$ & $q_{1}^{3}$ & $q_{2} q_{1}^{3}$ & $q_{2} q_{1}^{2}$ & $q_{2}^{2} q_{1}^{2}$ & $q_{2}^{2} q_{1}^{3}$ \\
\hline$\vdots$ & $\vdots$ & $\vdots$ & $\vdots$ & . & $\vdots$ \\
\hline $\begin{array}{c}q_{1}^{\alpha_{1}-2} \\
q_{1}^{\alpha_{1}}\end{array}$ & $q_{1}^{\alpha_{1}-1}$ & $q_{2} q_{1}^{\alpha_{1}-1}$ & $\begin{array}{c}q_{2} q_{1}^{\alpha_{1}-2} \\
q_{2} q_{1}^{\alpha_{1}}\end{array}$ & $\begin{array}{c}q_{2}^{2} q_{1}^{\alpha_{1}-2} \\
q_{2}^{2} q_{1}^{\alpha_{1}}\end{array}$ & $q_{2}^{2} q_{1}^{\alpha_{1}-1}$ \\
\hline
\end{tabular}

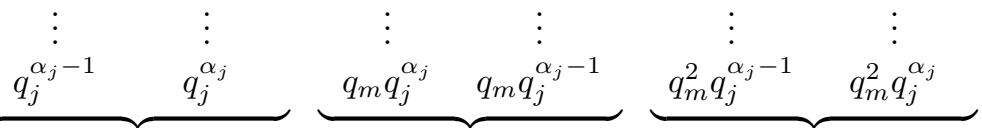

Extending the process for all prime divisors $q_{i}$, we obtain that $D_{1}\left(\prod_{i} q_{i}{ }^{\alpha_{i}}\right)=0$ if $n$ is not a perfect square.

We make this kind of list also if $n$ is a perfect square. Since all $\alpha_{i}$ are even, we have the following table: 
In the first two columns the number of divisors $\equiv 1(\bmod 3)$ exceeds the number of divisors $\equiv-1(\bmod 3)$ by one, whereas in every other block of four columns their numbers are equal (all other divisors can be grouped into such blocks of four columns, since the exponent of every prime is even).

It means that $D_{1}\left(\prod_{i} q_{i}{ }^{\alpha_{i}}\right)=1$ if $n$ is a perfect square.

TheOrem 1. If $n=\underbrace{\prod_{i} q_{i}{ }^{\alpha_{i}}}_{A} \cdot \underbrace{\prod_{j} p_{j}{ }^{\beta_{j}}}_{B}$, where $q_{i} \equiv-1(\bmod 3), p_{j} \equiv 1$ $(\bmod 3)$, then

$$
D_{1}(n)= \begin{cases}0 & \Leftrightarrow A \text { is not a perfect square } \\ d(B) & \Leftrightarrow A \text { is a perfect square }\end{cases}
$$

where $d(B)$ is the number of divisors of $B$.

Proof. This follows from Lemma 1 and Lemma 3.

Theorem 1 implies that the equation $D_{1}(n)=k$ can be solved for any $k \in \mathbb{Z}^{+}$. Furthermore, it has infinitely many solutions in $n$ for any given $k \in \mathbb{Z}^{+}$.

Next we study $R_{1}(n)$.

Theorem 2. If $n=\prod q_{i}^{\alpha_{i}} \cdot \prod p_{j}{ }^{\beta_{j}}$, where $q_{i} \equiv-1(\bmod 3), p_{j} \equiv 1$

$(\bmod 3)$, then

$$
R_{1}(n)= \begin{cases}1 & \Leftrightarrow A \text { is not a perfect square; } \\ \frac{d(A)-1}{d(A)+1} \Leftrightarrow A \text { is a perfect square. }\end{cases}
$$

Proof. If $A$ is not a perfect square, then $D_{1}(n)=0$ by Theorem 1 , which is equivalent to $R_{1}(n)=1$ by Lemma 2 .

If $A$ is a perfect square, then $\sum_{\substack{x \mid A \\ x \equiv 1(3)}} 1-\sum_{\substack{x \mid A \\ x \equiv-1(3)}} 1=1$ by Lemma 3 , and obviously

$$
\sum_{\substack{x \mid A \\ x \equiv 1(3)}} 1+\sum_{\substack{x \mid A \\ x \equiv-1(3)}} 1=d(A)
$$


hence

$$
\sum_{\substack{x \mid A \\ x \equiv-1(3)}} 1=\frac{d(A)-1}{2}
$$

and

$$
\sum_{\substack{x \mid A \\ x \equiv 1(3)}} 1=\frac{d(A)+1}{2} .
$$

As a corollary, we obtain $\frac{1}{2} \leq R_{1}(n) \leq 1$ for every $n \in \mathbb{Z}^{+}$.

Comparing the sum of divisors in different residue classes

Let $f(x)=x$, i.e. we examine the ratio $R_{i d}(n)$ and the difference $D_{i d}(n)$ of the sum of divisors $d$ of $n$ for which $d \equiv \pm 1(\bmod 3)$.

then

Lemma 4. If $n=\prod_{i} q_{i}{ }^{\alpha_{i}} \cdot \prod_{j} p_{j}{ }^{\beta_{j}}$, where $q_{i} \equiv-1(\bmod 3), p_{j} \equiv 1(\bmod 3)$,

$$
D_{i d}(n)=\prod_{j} \frac{p_{j}^{\beta_{j}+1}-1}{p_{j}-1} \cdot \prod_{i} \frac{\left(-q_{i}\right)^{\alpha_{i}+1}-1}{\left(-q_{i}\right)-1} .
$$

Proof. If we have a divisor $d$ of form $d=\prod_{i}{q_{i}}^{\mu_{i}} \cdot \prod_{j} p_{j}{ }^{\nu_{j}}$, then $d \equiv$ $1(\bmod 3)$ iff $\sum_{i} \mu_{i}$ is even, and $d \equiv-1(\bmod 3)$ iff $\sum_{i} \mu_{i}$ is odd. Therefore $D_{i d}(n)$ can be written as the product of geometric series:

$$
\begin{gathered}
D_{i d}(n)= \\
=\prod_{j}\left(1+p_{j}+p_{j}^{2}+p_{j}^{3}+\ldots+p_{j}^{\beta_{j}}\right) \cdot \prod_{i}\left(1-q_{i}+q_{i}^{2}-q_{i}^{3}+\ldots+(-1)^{\alpha_{i}} q_{i}^{\alpha_{i}}\right) .
\end{gathered}
$$

Using the summation formula, we obtain the desired result.

We note that the same argument can be applied for any multiplicative (non zero) function $f$ : 


$$
\begin{gathered}
D_{f}(n)=\prod_{j}\left(1+f\left(p_{j}\right)+f\left(p_{j}^{2}\right)+f\left(p_{j}^{3}\right)+\ldots+f\left(p_{j}^{\beta_{j}}\right)\right) . \\
\cdot \prod_{i}\left(1-f\left(q_{i}\right)+f\left(q_{i}^{2}\right)-f\left(q_{i}^{3}\right)+\ldots+(-1)^{\alpha_{i}} f\left(q_{i}^{\alpha_{i}}\right)\right) .
\end{gathered}
$$

Applying this for $f(x)=1$, we obtain a new proof for Theorem 1 .

Also, if $f$ is completely multiplicative, i.e. $f(a b)=f(a) f(b)$ for every $a, b$, and $f(p) \neq 1$ for any prime $p$, then

$$
D_{f}(n)=\prod_{j} \frac{f\left(p_{j}\right)^{\beta_{j}+1}-1}{f\left(p_{j}\right)-1} \cdot \prod_{i} \frac{\left(-f\left(q_{i}\right)\right)^{\alpha_{i}+1}-1}{\left(-f\left(q_{i}\right)\right)-1} .
$$

Now we show that there is at least one value missing from the range of $R_{i d}(n)$.

Theorem 3.

$$
\forall n \in \mathbb{Z}^{+} \quad R_{i d}(n) \neq 1 \text {. }
$$

Proof. $R_{i d}(n) \neq 1 \Leftrightarrow D_{i d}(n) \neq 0$ by Lemma 2. By Lemma $4, D_{i d}(n)$ is a product where the (numerators of the) factors cannot be zero, hence $D_{i d}(n) \neq 0$.

We give another proof for Theorem 3 if $A$ is squarefree $(n=\underbrace{\prod_{i=1}^{m} q_{i}}_{A} \cdot \prod_{j} p_{j}^{\beta_{j}})$.

Proof. We have

$$
R_{i d}(n) \neq 1 \Leftrightarrow D_{i d}(n) \neq 0 \Leftrightarrow D_{i d}(A) \neq 0 \Leftrightarrow \sum_{\substack{x \mid A \\ x \equiv-1(3)}} x-\sum_{\substack{x \mid A \\ x \equiv 1(3)}} x \neq 0,
$$

which is equivalent to

$$
\sigma(A) \neq 2 \cdot \sum_{\substack{x \mid A \\ x \equiv 1(3)}} x \Leftrightarrow \prod_{i=1}^{m}\left(1+q_{i}\right) \neq 2 \cdot\left(1+\sum_{i, j} q_{i} q_{j}+\sum_{i, j, k, l} q_{i} q_{j} q_{k} q_{l}+\ldots\right),
$$

where $\sigma(A)$ denotes the sum of divisors of $A$. The last inequality follows from

$$
3 \mid \prod_{i=1}^{m}\left(1+q_{i}\right)
$$


while

$$
\begin{gathered}
2 \cdot\left(1+\sum_{i, j} q_{i} q_{j}+\sum_{i, j, k, l} q_{i} q_{j} q_{k} q_{l}+\ldots\right) \equiv 2 \cdot\left(1+\left(\begin{array}{c}
m \\
2
\end{array}\right)+\left(\begin{array}{c}
m \\
4
\end{array}\right)+\ldots\right)= \\
=2 \cdot 2^{m-1}=2^{m} \not \equiv 0(\bmod 3) .
\end{gathered}
$$

The argument above proves also the following statement: If $n=\prod_{i=1}^{m} q_{i}$ and $R_{i d}(n)=c$ for some $c \in \mathbb{Z}^{+}$, then $c \equiv-1\left(\bmod 3^{m}\right)$.

Now we characterize how the values of $R_{i d}(n)$ and $D_{i d}(n)$ are distributed in the ranges.

\section{THEOREM 4.}

$$
R_{i d}(n)>1 \Leftrightarrow D_{i d}(n)<0 \Leftrightarrow n \equiv-1(\bmod 3) .
$$

Proof. The first equivalence is contained in Lemma 2. As for the second one, Lemma 4 implies that $D_{i d}(n)<0$ iff there is an odd number of negative factors in the product, or equivalently there is an odd number of numerators in which $\alpha_{i}$ is odd, i.e. $\sum_{i} \alpha_{i}$ is odd which means $n \equiv-1(\bmod 3)$.

Now we show that $R_{i d}(n)$ can assume arbitrarily large and arbitrarily small positive values, as well.

\section{THEOREM 5.}

$$
\forall K \in \mathbb{R}^{+} \quad \exists n \in \mathbb{Z}^{+} \text {such that } R_{i d}(n)>K
$$

Proof. If $n=q^{2 \alpha+1}$ with a prime $q \equiv-1(\bmod 3)$ and $\alpha \in \mathbb{N}$, then $R_{i d}(n)=q$, and there exist infinitely many such primes.

Moreover, there are infinitely many $c \in \mathbb{R}^{+}$for which $R_{i d}(n)=c$ has infinitely many solutions in $n$ : for any prime $c \equiv-1(\bmod 3), R_{i d}(n)=c$ has infinitely many solutions $n=c^{2 \alpha+1}, \alpha \in \mathbb{N}$.

\section{THEOREM 6.}

$$
\forall k \in \mathbb{R}^{+} \quad \exists n \in \mathbb{Z}^{+} \text {such that } R_{i d}(n)<\frac{1}{k} .
$$


Proof. If $n=q^{2 \alpha}$ with a prime $q \equiv-1(\bmod 3)$ and $\alpha \in \mathbb{Z}^{+}$, then

$$
R_{i d}(n)=\frac{q+q^{3}+\ldots+q^{2 \alpha-1}}{1+q^{2}+\ldots+q^{2 \alpha}}<\frac{1}{q} .
$$

Comparing the sum of reciprocals of divisors in different residue classes

Let $f(x)=\frac{1}{x}$, so we study the ratio $R_{r e c}(n)$ and the difference $D_{\text {rec }}(n)$ of the sum of reciprocals of divisors $d$ of $n$ for which $d \equiv \pm 1(\bmod 3)$.

\section{THEOREM 7.}

$$
R_{r e c}(n)= \begin{cases}R_{i d}(n) & \Leftrightarrow n \equiv 1(3) ; \\ \frac{1}{R_{i d}(n)} & \Leftrightarrow n \equiv-1(3) .\end{cases}
$$

PROOF. Using the complementary divisors we have

$$
R_{r e c}(n)=\frac{\sum_{\substack{x \mid n \\ x \equiv-1(3)}} \frac{1}{x}}{\sum_{\substack{y \mid n \\ y \equiv 1(3)}} \frac{1}{y}}=\frac{\sum_{\substack{x, x,=n \\ x \equiv-1(3)}} \frac{x}{n}}{\sum_{\substack{y \cdot y,=n \\ y \equiv 1(3)}} \frac{y^{\prime}}{n}}=\frac{\sum_{\substack{x \cdot x,=n \\ x \equiv-1(3)}} x}{\sum_{\substack{y \cdot y,=n \\ y \equiv 1(3)}} y^{\prime}} .
$$

If $n \equiv 1(\bmod 3)$, then $x^{\prime} \equiv x$ and $y^{\prime} \equiv y(\bmod 3)$, hence

$$
\sum_{\substack{x \cdot x,=n \\ x \equiv-1(3)}} x^{\prime}=\sum_{\substack{x \mid n \\ x \equiv-1(3)}} x \text { and } \sum_{\substack{y \cdot y,=n \\ y \equiv 1(3)}} y^{\prime}=\sum_{\substack{y \mid n \\ y \equiv 1(3)}} y, \text { so } R_{r e c}(n)=R_{i d}(n) .
$$

If $n \equiv-1(\bmod 3)$, then $x^{\prime} \equiv 1$ and $y^{\prime} \equiv-1(\bmod 3)$, so $\sum_{\substack{x \cdot x,=n \\ x \equiv-1(3)}} x=\sum_{\substack{y \mid n \\ y \equiv 1(3)}} y$ and $\sum_{\substack{y \cdot y,=n \\ y \equiv 1(3)}} y^{\prime}=\sum_{\substack{x \mid n \\ x \equiv-1(3)}} x$, which implies $R_{r e c}(n)=\frac{1}{R_{i d}(n)}$

By the same arguments, we obtain also 
THEOREM 8.

$$
D_{r e c}(n)= \begin{cases}\frac{D_{i d}(n)}{n} & \Leftrightarrow n \equiv 1(3) ; \\ -\frac{D_{i d}(n)}{n} & \Leftrightarrow n \equiv-1(3) .\end{cases}
$$

We note that Theorem 8 follows also by the method sketched after the proof of Lemma 4.

For the ranges we have the following result:

Theorem 9. $R_{\text {rec }}(n)<1$ and $D_{\text {rec }}(n)>0$ for every $n \in \mathbb{Z}^{+}$.

Proof. Theorems 4 and 7 clearly imply $R_{r e c}(n)<1$. Theorems 4 and 8 yield immediately $D_{r e c}(n)>0$. (Also, the two statements are equivalent by Lemma 2.)

We give another proof if $A$ is squarefree $(n=\underbrace{\prod_{i} q_{i}}_{A} \cdot \prod_{j} p_{j}^{\beta_{j}})$.

Proof. Clearly, $D_{\text {rec }}(n)>0 \Leftrightarrow D_{\text {rec }}(A)>0$, and

$$
D_{\text {rec }}(A)=1-\sum_{i} \frac{1}{q_{i}}+\sum_{i, j} \frac{1}{q_{i} q_{j}}-\sum_{i, j, k} \frac{1}{q_{i} q_{j} q_{k}}+\sum_{i, j, k, l} \frac{1}{q_{i} q_{j} q_{k} q_{l}}-\ldots=\frac{\varphi(A)}{A}>0
$$

(where $\varphi(n)$ is Euler's totient function).

We note that the second proof can be extended also for the case when $A$ is not squarefree, but the proof is technically a bit more difficult.

\section{Didactical remarks}

We mention here a few ideas why and how the investigations in this topic can help secondary school students to improve their mathematical horizon and abilities.

a) The art of generalization. Starting from simple observations, students can make natural generalizations themselves. E.g. after solving our initial question about the ratio of the sums of even and odd divisors, we can change the modulus from 2 to 3 , and get two different types of problems. The first one 
when even/odd is replaced by divisibile/non-divisible by 3 does not require any new ideas. But the second one, when we classify the divisors according to their residues +1 or -1 mod 3 , is much tougher. Another generalization is if, instead of the sum of divisors, we study various other functions of divisors, and students can figure out such functions themselves. This way they experience how to develop and extend a question and how to ask new ones. Moreover, students can also realize that the common essential feature of these functions was their multiplicative property.

b) Conjecturing from examples calculated using mathematical software. Especially GeoGebra or WolframAlpha can be useful aids. The first step and challenge is to create a suitable tool from the given functions of the software to study the questions, which requires a good amount of willingness to experiment and mathematical thinking for sure. A nice example is, if we compare the sums of divisors in the three nonzero residue classes mod 4. Calculating these three sums for sufficiently many values of $n$, sooner or later most students will discover the following relation:

$$
2 \cdot \frac{\sum_{d \equiv 3(4)} d}{\sum_{d \equiv 1(4)} d}+2=\frac{\sum_{d \equiv 2(4)} d}{\sum_{d \equiv 1(4)} d} .
$$

And - after having the formula - it is not too hard to prove this conjecture:

$$
2 \cdot \frac{\sum_{\substack{d \mid n \\ d \equiv-1(4)}} d}{\sum_{\substack{d \mid n \\ d \equiv 1(4)}} d}+2=2 \cdot\left(\frac{\sum_{\substack{d \mid n \\ \equiv=-1(4)}} d}{\sum_{\substack{d \mid n \\ d \equiv 1(4)}} d}+1\right)=2 \cdot\left(\frac{\sum_{\substack{d \mid n \\ d \equiv-1(4)}} d+\sum_{\substack{d \mid n \\ d \equiv=1(4)}} d}{\sum_{\substack{d \mid n \\ d \equiv 1(4)}} d}\right),
$$

and clearly,

$$
2 \cdot\left(\sum_{\substack{d \mid n \\ d \equiv-1(4)}} d+\sum_{\substack{d \mid n \\ d \equiv 1(4)}} d\right)=\sum_{\substack{d \mid n \\ d \equiv 2(4)}} d .
$$

c) The grades of difficulty - easy, hard, and unsolved - through the variants of a problem. We saw that we could easily solve some of the problems, but modifying them the new variant was sometimes much harder. And even the students can easily ask some natural questions for which we do not know the answer at present. E.g. we have proved that $R_{i d}(n)$ never equals 1 , but we do 
not know if there is any other positive value missing from the range of $R_{i d}(n)$. Are the primes $q \equiv-1(\bmod 3)$ the only integer values in the range of $R_{i d}(n)$ ? Or, to be more modest, is it true that if $n$ has two distinct prime divisors $\equiv-1(\bmod 3)$, then $R_{i d}(n) \notin \mathbb{Z}$ ? Also, if we change the modulus from 3 to 4 , and examine the distribution of the divisors in the reduced residue classes, we can prove analogous statements exactly the same way as we did mod 3. But having 5 as modulus, we do not even know whether $R_{i d}(n)$ can be equal to 1. Since there are four nonzero residue classes $\bmod 5$, the study of $D_{i d}(n)$ is much more difficult in this case than it was mod 3. These kinds of problems can help students to get used to the fact that many innocent looking natural questions can lead to unsolved problems, and this is a natural phenomenon in mathematics.

d) The variety of methods. Giving different proofs for a theorem, we get a deeper insight and find bridges between the various branches of mathematics, e.g. between number theory and combinatorics as in the second proofs of Theorems 3 and 9 . This way children can realize that mathematics is not just a summation of separate, independent fields, but these fields are strongly connected to each other.

I am very grateful for the help and suggestions of my supervisor, Róbert Freud and for the referees' valuable remarks.

\section{References}

[1] R. Freud and E. Gyarmati, Számelmélet, in Hungarian, Nemzeti Tankönyvkiadó, Budapest, 2000.

[2] I. Niven, H. S. Zuckerman and H. L. Montgomery, An Introduction to the Theory of Numbers, 5th edition, John Wiley and Sons Inc., New York, 1991.

ILDIKÓ KÉZÉR

ÓBUDAI ÁRPÁD GIMNÁZIUM

BUDAPEST

HUNGARY

E-mail: kezerildi@gmail.com

(Received September, 2016) 